\title{
Abnormal neural response to feedback on planning and guessing tasks in patients with unipolar depression
}

\author{
R. ELLiOTT, ${ }^{1}$ B. J. SAHAKIAN, A. MiCHAEL, E. S. PAYKEL AND R. J. DOLAN \\ From the Wellcome Department of Cognitive Neurology, Institute of Neurology and Royal Free Hospital \\ School of Medicine, London; and Department of Psychiatry, University of Cambridge
}

\begin{abstract}
Background. It has been suggested that patients with unipolar depression show abnormal responses to negative feedback in the performance of cognitive tasks. Positron emission tomography (PET) has previously identified blood flow abnormalities in depressed patients during cognitive performance. We have also used PET to identify regions where there is differential neural response to performance feedback in normal volunteers. In this study we aimed to test the hypothesis that blood flow in these regions, the medial caudate and ventromedial prefrontal cortex, would be abnormal in depressed patients.
\end{abstract}

Methods. Six patients with unipolar depression and six matched controls were scanned using PET while performing cognitive tasks in the presence and absence of feedback.

Results. Compared with controls, depressed patients failed to show significant activation in the medial caudate and ventromedial orbitofrontal cortex. Blood flow was lower and a differential response, observed in normals, under different task and feedback conditions was not seen in the patients.

Discussion. The findings suggest that the behavioural response to feedback in depressed patients is associated with an abnormal neural response within the medial caudate and ventromedial orbitofrontal cortex, regions implicated in reward mechanisms. We argue that the observed abnormalities may depend on a combination of psychological factors, with both cognitive and emotive components.

\section{INTRODUCTION}

Influential cognitive and behavioural accounts of depression have proposed a key role for reinforcement in its onset and maintenance. For example, Lewinsohn (1974; Lewinsohn et al. 1979) proposed a behavioural model of depression based on reinforcement which hypothesized that various reinforcement-reducing contingencies may apply in depressed patients. Thus, they find fewer events potentially reinforcing, experience fewer of those events which they do find reinforcing and receive reduced social

1 Address for correspondence: Dr Rebecca Elliott, Wellcome Department of Cognitive Neurology, 12 Queen Square, London WC1N 3BG. reinforcement due to impaired social skills. In the depressed state opportunities for social, and other forms of, reinforcement are typically reduced, which further exacerbates the effects of an already abnormally low rate of reinforcement. The concept of feedback response can also be linked to cognitive accounts of the disorder. An influential cognitive theory, developed by Beck (1967) and subsequently modified by Teasdale (1988), argues that individuals vulnerable to depression are characterized by enduring dysfunctional schemata made up of negative attitudes and assumptions. A bias towards the processing of negative reinforcement may detract from their ability to perform normally in response to cognitive challenges.

Reinforcement, in the form of performance 
feedback, is a key component in many laboratory tests of cognitive function. Performance feedback has both evaluative and emotive components; it can be used to guide and monitor performance but also has the emotional consequences of being correct and incorrect. Depression has reliably been shown to be associated with impairments on tests of cognitive function (e.g. Weingartner et al. 1981; Austin et al. 1992; Brown et al. 1994; Elliott et al. 1996) and recent evidence indicates a deficit in the response to performance feedback. For example, Hughes et al. (1985) showed that depressed patients were less responsive to reinforcement than controls. Using a progressive schedule for financial reward, they found that depressed subjects worked less for reward and so earned less. Similarly, Henriques et al. (1994) compared response biases under a neutral and a rewarding pay-off condition and showed that dysphoric subjects showed less difference between these conditions than a non-dysphoric group. There is also evidence to suggest that depressed subjects evaluate their performance in a more negative way than controls. Specifically, Wener \& Rehm (1975) found that depressed patients reported a lower self-assessed success rate than controls.

Two recent studies suggest that depressed patients are particularly sensitive to negative feedback during neuropsychological task performance. Beats et al. (1996) proposed a 'catastrophic response to executive failure' to explain the rapid deterioration in planning performance of elderly depressed patients after making an error. This catastrophic response to failure was tested more explicitly by Elliott et al. (1996) who suggested that patients with unipolar depression showed a greater sensitivity to negative feedback than controls, assessed in terms of its detrimental effect on subsequent performance. Abnormal response to negative feedback has also been shown to be specific to depression and related to clinical severity (Elliott et al. 1997). This study reformulated the "catastrophic response to failure' argument somewhat, suggesting that the discrepancy between depressed patients and controls may also be due to control subjects responding to negative feedback by augmenting performance on subsequent trials. Clearly though, these studies report an abnormality in the behavioural responses of depressed patients to feedback.
These behavioural effects can be seen as an important link between depressed mood and cognitive performance in depression. The present study sought to identify a specific neural substrate for this effect. A recent functional imaging study in normal volunteers (Elliott et al. 1997) considered the modulation of cognitive task-related activations by feedback. Regions sensitive to the presence, compared with absence, of feedback were the medial caudate nucleus and the ventromedial orbitofrontal cortex. Significantly greater activation in these structures was seen during a guessing task compared to a planning task. The striatal and cortical loci observed in this study are entirely congruent with animal and human data, which indicate that processing of performance feedback is associated with the ventral striatum (incorporating the medial caudate) and orbitofrontal cortex (e.g. Thorpe et al. 1983; Schultz et al. 1992; Bechara et al. 1994; Rolls et al. 1994).

Previous PET studies have demonstrated abnormal prefrontal blood flow in depressed patients (e.g. Cohen et al. 1989; Bench et al. 1992; Drevets et al. 1992) that may relate to clinical features of the disorder. It should be noted however, that the reported prefrontal abnormalities in these studies did not include the ventromedial orbitofrontal cortex, which may have been outside the field of view of early imaging techniques. More recent studies have reported orbitofrontal abnormalities in depressed patients (Mayberg et al. 1994, 1997; Drevets et al. 1997) and therefore it is reasonable to hypothesize abnormalities in this region. Findings of reduced activity in the caudate nucleus of depressed patients in the resting state have also been reported (Buchsbaum et al. 1986; Cohen et al. 1989; Drevets et al. 1992). Further, a recent cognitive activation study of patients performing a planning task has indicated functional abnormalities in prefrontal structures as well as the caudate nucleus (Elliott et al. 1997).

The present study used the paradigm developed in our recent study of normal volunteers (Elliott et al. 1997) to determine whether the reported abnormal behavioural response to performance feedback in depressed patients could be related to abnormal neural function. Specifically, we hypothesized that in depression, the presence of performance feedback would be associated with an abnormal neural response in 
the ventral striatum and orbitofrontal cortex, structures we conjecture to be important substrates of reinforcement mediated behaviour.

\section{METHOD \\ Subjects}

Six right-handed male patients with a diagnosis of recurrent major depressive disorder (DSM-IV, APA, 1994)) were recruited for this study. Patients with history of neurological disorder, head injury or substance abuse were excluded as were patients who had received ECT in the preceding 12 months. The mean age of the patients was 41 (range 24-54). The severity of their depression was assessed using the 17item Hamilton Depression Scale (HAM-D, Hamilton, 1960); mean score 26.0 (range 19-33): and the Montgomery-Asberg Depression Rating Scale (MADRS, Montgomery \& Åsberg, 1979); mean score 34.7 (range 28-38). All of the patients were on antidepressant medication; one on $100 \mathrm{mg}$ atenolol $+210 \mathrm{mg}$ lofepramine, one on $30 \mathrm{mg}$ paroxetine, one on $30 \mathrm{mg}$ paroxetine + lithium, one on $300 \mathrm{mg}$ venlafaxine $+15 \mathrm{mg}$ zopiclone, one on $200 \mathrm{mg}$ imipramine and one on $200 \mathrm{mg}$ sertraline.

These patients were compared with the six right-handed male control volunteers (mean age 35 ) scanned in our previous study (Elliott et al. 1997). The groups were matched for age and educational attainment. As this study used false feedback, all subjects were thoroughly debriefed after their participation. The study was approved by the Addenbrooke's Hospital Local Research Ethics Committees and the Advisory Committee on the Administration of Radioactive Substances (ARSAC) UK. All subjects gave informed consent.

\section{Cognitive activation paradigm}

The design of this experiment was a two by two by three factorial design with subject group (two levels; patients and controls) as one factor, experimental task (two levels; planning and guessing) as another, and feedback condition (three levels; positive, negative and no feedback) as the third.

\section{Planning task}

This task was based on the Tower of London task (Shallice, 1982), using the one-touch ap- proach developed by Owen et al. (1995). The present version was the variant of this paradigm, used in an earlier study of normal volunteers (Elliott et al. 1997). Subjects were presented two arrays of coloured balls and asked to work out the minimum number of moves needed to make one array match the other, according to rules which were explained before scanning. Ten seconds were allowed, after which the arrays disappeared and subjects had to press one of six labelled buttons that corresponded to their solution. Problems were all of three to six moves and subjects typically require in the region of $15-25 \mathrm{~s}$ to solve problems of this difficulty (Owen et al. 1995; Elliott et al. 1996) when no time constraint is imposed. The time limit therefore prevented subjects being certain of their answers, thus rendering them more likely to believe false feedback. Ten unique problems were presented during each scan, the sequence starting $60 \mathrm{~s}$ prior to scanning to ensure the establishment of the appropriate cognitive set. Subjects were explicitly told to optimize performance before the start of each scan.

\section{Guessing task}

Subjects were presented with two identical arrays of coloured balls and asked to watch until they disappeared (after a mean of $10 \mathrm{~s}$ ). This was a signal for them to press one of the six response buttons. They were told that on each trial, three of the buttons would be randomly assigned as correct. Subjects were told before each sequence that this assignment was purely chance so that each response constituted a 50:50 guess. Again 10 problems were performed during each scanning period, starting $60 \mathrm{~s}$ before scanning.

\section{Feedback conditions}

Both the above tasks were performed under three different conditions. Feedback was given after every trial and constituted a large green tick and the words 'YOU ARE RIGHT' or a large red cross and the words 'YOU ARE WRONG', presented on the screen for $1 \mathrm{~s}$ immediately after each response. In the positive feedback condition, subjects were told they were correct on every trial, regardless of their actual performance. In the negative condition, subjects were told they were wrong on $80 \%$ of trials, regardless of whether their responses were actually correct. In the neutral condition, no 
feedback was given. Between trials subjects saw a display comprising a large triangle and the words 'PLEASE WAIT'. Each of the six conditions in this factorial design was performed twice in a fully counterbalanced order within and between subjects.

\section{PET scanning technique}

An ECAT HR + scanning system (CTI Siemens, Knoxville, TN) was used to measure regional cerebral blood flow (rCBF). For each scan, $555 \mathrm{Mbq}$ of $\mathrm{H}_{2}{ }^{15}[\mathrm{O}]$ was flushed with normal saline through a cannula in the left antecubital vein over $20 \mathrm{~s}$ at a rate of $10 \mathrm{ml} / \mathrm{min}$ by an automatic pump. After a delay of approximately $35 \mathrm{~s}$, a rise in counts could be detected at the head. This peaked $30-40 \mathrm{~s}$ later, the exact interval depending on individual subjects. Data were acquired during a single $90 \mathrm{~s}$ frame, beginning $5 \mathrm{~s}$ before the head curve began to rise. Twelve scans were performed in all at $8 \mathrm{~min}$ intervals. A transmission scan with an exposed $68 \mathrm{Ge} / 68 \mathrm{Ga}$ external ring source was performed before each session to correct for attenuation. Filtered back projection was used to reconstruct the images, giving a resolution of $6.0 \mathrm{~mm}$ at full width half maximum and displayed in a $128 \times 128$ pixel format with 63 planes rendering the voxels approximately cubic.

\section{Data analysis}

Statistical Parametric Mapping (SPM96, Wellcome Department of Cognitive Neurology, London, UK) was used to analyse the data implemented in MATLAB (Mathworks, Inc., Sherborn, MA, USA) and run on a SPARC workstation (Sun Microsystems Inc., Surrey, UK). Scans were realigned using the first as a reference and were then transformed into the standard space of Talairach \& Tournoux (1988) using MNI templates (Montreal Neurological Institute). Images were smoothed with a $16 \mathrm{~mm}$ full width half maximum Gaussian filter.

Conditions for each subject were specified in the appropriate design matrix, which included global activity as a confounding covariate, so this can be considered an ANCOVA. Effects at each and every voxel were estimated according to the general linear model and regionally specific effects were compared using linear contrasts. The resulting set of voxel values for each contrast constituted a statistical parametric map of the $t$

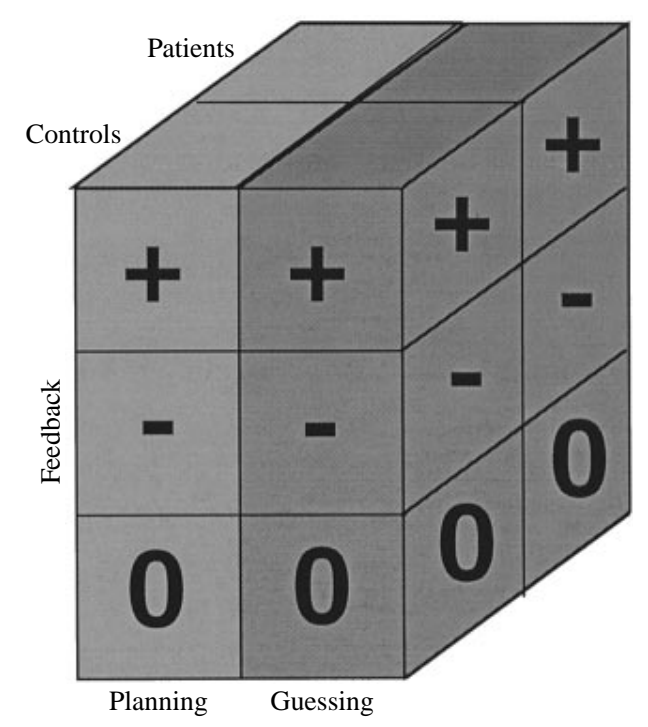

FIG. 1. Graphical depiction of the $2 \times 2 \times 3$ factorial study design.

statistic $(\operatorname{SPM}\{t\})$ which was then transformed to the unit normal distribution, $\operatorname{SPM}\{Z\}$ (Friston et al. 1995).

The design of this experiment was a $2 \times 2 \times 3$ factorial with subject group as one factor, task as another and feedback type as the third (see Fig. 1). In the analysis of these data, the critical questions were which regions of those activated in normal subjects in association with performance feedback showed abnormal activation in depressed patients. Our earlier study had identified the medial caudate and ventromedial prefrontal cortex as the key foci and we hypothesized that abnormalities in these regions would be seen in depression. Since specific neuroanatomical hypotheses are being considered, we report uncorrected $P$ values with respect to predicted regions of difference. In PET studies where there is no anatomical hypothesis, it is necessary to correct $P$ values for multiple comparisons since comparisons are being made at every voxel and there is therefore a high chance of Type 1 errors. In this study, we were hypothesizing focal abnormalities in two distinct regions; the medial caudate and the ventromedial orbitofrontal cortex.

Given that this is an anatomically constrained hypothesis, the issue of correction for multiple comparisons is not of the sort usually encountered in SPM analyses of this type. Hitherto, one would normally have accepted a threshold 
Table 1. Co-ordinates of maximal significant change in $r C B F$ associated with the key feedbackrelated comparisons in the normal subjects only (see Elliott et al. 1997)

\begin{tabular}{|c|c|c|c|c|c|c|}
\hline \multirow[b]{3}{*}{$\mathrm{rCBF}$ increases } & \multicolumn{6}{|c|}{ Region of activation } \\
\hline & \multirow[b]{2}{*}{ Left/Right } & \multirow{2}{*}{$\begin{array}{c}\text { Brodmann's } \\
\text { area }\end{array}$} & \multicolumn{3}{|c|}{ Talairach co-ordinates } & \multirow{2}{*}{$\begin{array}{c}Z \text { value } \\
(3.09 \text { for } \\
P<0.001)\end{array}$} \\
\hline & & & $x$ & $y$ & $z$ & \\
\hline \multicolumn{7}{|l|}{ Feedback present-absent } \\
\hline \multirow[t]{2}{*}{ Medial caudate nucleus } & $\mathrm{R}$ & & 8 & 16 & 16 & 3.67 \\
\hline & $\mathrm{L}$ & & -10 & 16 & 4 & $3 \cdot 56$ \\
\hline \multirow{2}{*}{ Superior temporal gyrus } & $\mathrm{L}$ & 22 & -32 & -34 & 4 & $3 \cdot 72$ \\
\hline & $\mathrm{R}$ & 38 & 42 & 8 & -8 & $3 \cdot 25$ \\
\hline \multicolumn{7}{|c|}{ Guessing-planning feedback present-absent } \\
\hline \multirow[t]{2}{*}{ Ventromedial orbitofrontal cortex } & $\mathrm{R}$ & 25 & 4 & 30 & -20 & $3 \cdot 61$ \\
\hline & $\mathrm{L}$ & 25 & -4 & 22 & -12 & $3 \cdot 18$ \\
\hline Anterior frontal cortex & $\mathrm{R}$ & 10 & 14 & 58 & -4 & $3 \cdot 35$ \\
\hline Inferior frontal gyrus & $\mathrm{L}$ & 47 & -28 & 22 & 0 & $4 \cdot 05$ \\
\hline \multicolumn{7}{|l|}{ Present-absent guessing only } \\
\hline Ventromedial orbitofrontal cortex & $\mathrm{R}$ & 11 & 10 & 30 & -20 & $3 \cdot 64$ \\
\hline Medial caudate nucleus & $\mathrm{R}$ & & 8 & 20 & 8 & $4 \cdot 08$ \\
\hline Inferior frontal gyrus & $\mathrm{L}$ & 47 & -22 & 22 & 0 & 3.96 \\
\hline Superior temporal gyrus & $\mathrm{R}$ & 38 & 40 & 8 & -12 & $3 \cdot 53$ \\
\hline
\end{tabular}

Table 2. Co-ordinates where $\mathrm{CBF}$ changes were seen in control subjects relative to depressed patients associated with comparisons of different feedback conditions

\begin{tabular}{|c|c|c|c|c|c|c|}
\hline \multirow{2}{*}{$\begin{array}{l}\text { Relative activations } \\
\text { presence-absence }\end{array}$} & \multicolumn{6}{|c|}{ Region of activation } \\
\hline & Left/Right & $\begin{array}{l}\text { Brodmann's } \\
\text { area }\end{array}$ & \multicolumn{3}{|c|}{ Talairach co-ordinates } & $\begin{array}{c}Z \text { value } \\
(3.09 \text { for } \\
P<0.001)\end{array}$ \\
\hline \multirow{2}{*}{ Medial caudate nucleus } & $\mathrm{R}$ & & 4 & 20 & 16 & $3 \cdot 18$ \\
\hline & $\mathrm{L}$ & & -4 & 16 & 4 & $2 \cdot 87$ \\
\hline
\end{tabular}

of $P<0.001$ (uncorrected) as being an adequate correction for an effect that was specified regionally but where the precise voxel was unknown. Recent advances in Gaussian Field Theory have now enabled us to provide exact critical values for small specified volumes (Worsley et al. 1996). Applying these results to the data reported in the current paper, they survive a correction for the small volume analysed.

For other regions, we have descriptively reported $P$ values at $P<0.001$ uncorrected, corresponding to $Z=3 \cdot 09$, although strong conclusions cannot be drawn about these regions in the absence of correction for multiple comparisons.

The stereotactic coordinates of Talairach \& Tournoux (1988) are used to report the observed activation foci. However, the descriptions of the anatomical localization of these foci were de- termined using the averaged structural MRIs of the group and the atlas of Duvernoy (1991). We have found that this method provides a more accurate localization than the Talairach \& Tournoux atlas (1988).

\section{RESULTS}

Within-group comparisons: normal volunteers

These findings have been reported in detail in our earlier paper (Elliott et al. 1997). The main points are summarized here because the regions of significant rCBF changes in normals are used to constrain our anatomical hypothesis about depressed patients. The critical comparisons in this study are those associated with different feedback conditions. The two feedback present conditions (positive and negative averaged) compared with the no feedback condition were associated with significant activations in the 
(a)
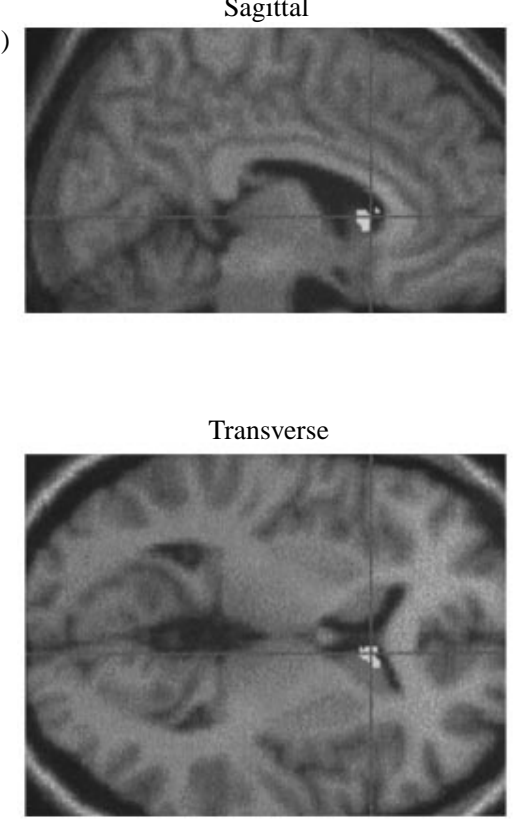

Coronal

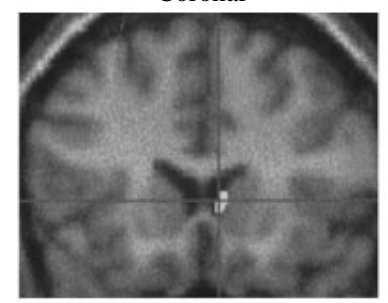

Talairach and Tournoux co-ordinates:

$x=4, y=20, z=16$

(b)

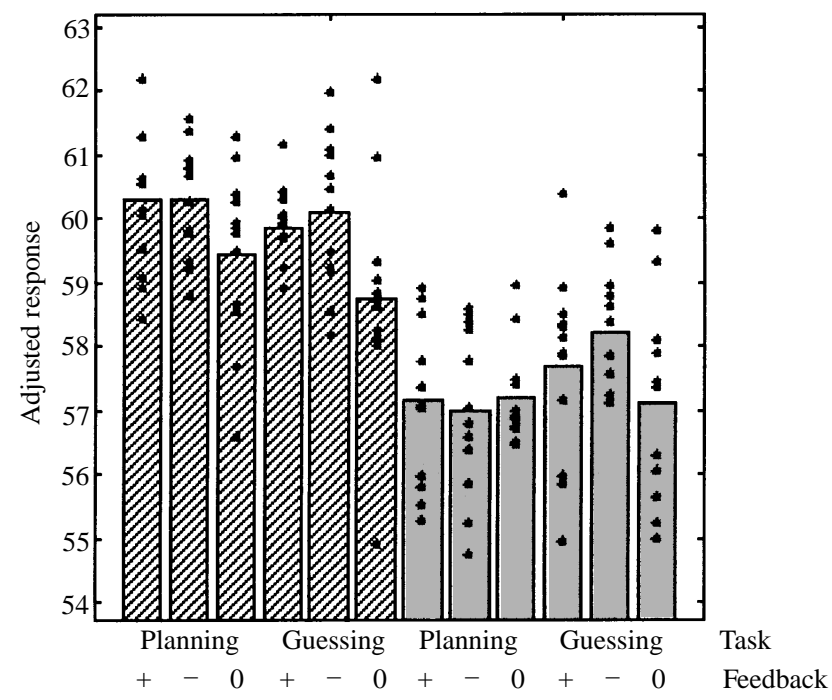

FIG. 2. Activations associated with the presence compared to the absence of feedback. (a) Shows the statistical parametric map of the $t$ statistic (after transformation to a SPM $Z Z$ ) thresholded at $P<0.01$ rendered onto a standard MRI template and focused on the medial caudate nucleus activation. (b) Shows absolute values of blood flow for the two subject groups ( $\square$, controls; 圆, patients) under the six experimental conditions in the medial caudate nucleus.

bilateral medial caudate nuclei and the right superior temporal gyrus (see Table 1). No significant deactivations were seen. Comparison between positive and negative feedback showed no significant $\mathrm{rCBF}$ differences and, therefore, the feedback conditions were combined in the interaction terms.

The modulation of task by feedback was 
Table 3. Co-ordinates where control subjects show significantly elevated $r C B F$ compared to depressed patients associated with the modulation of guessing compared to planning performance by different feedback conditions

\begin{tabular}{|c|c|c|c|c|c|c|}
\hline \multirow{3}{*}{$\begin{array}{l}\text { Relative activations } \\
\text { presence-absence }\end{array}$} & \multicolumn{6}{|c|}{ Region of activation } \\
\hline & \multirow[b]{2}{*}{ Left/Right } & \multirow{2}{*}{$\begin{array}{l}\text { Brodmann's } \\
\text { area }\end{array}$} & \multicolumn{3}{|c|}{ Talairach co-ordinates } & \multirow{2}{*}{$\begin{array}{c}Z \text { value } \\
\text { (3.09 for } \\
P<0.001)\end{array}$} \\
\hline & & & $x$ & $y$ & $z$ & \\
\hline \multirow[t]{2}{*}{ Ventromedial orbitofrontal cortex } & $\mathrm{L}$ & 25 & -6 & 22 & -16 & $2 \cdot 57$ \\
\hline & $\mathrm{R}$ & 25 & 4 & 30 & -16 & $2 \cdot 36$ \\
\hline
\end{tabular}

associated with significant activation in the ventromedial orbitofrontal cortex (BA 11/25), anterior frontal cortex (BA 10) and left inferior frontal gyrus (BA 47). These activations were associated with greater activity in the presence of feedback in the guessing compared to the planning task. The significant interaction was characterized further by considering the simple main effects of feedback in the two asks separately. There were no significantly increased activations associated with the presence compared to the absence of feedback in the planning task. In the guessing task, significant increases in activation were observed in the medial caudate nucleus extending rostrally to the ventral orbitofrontal cortex and in the left inferior frontal gyrus (BA 47).

\section{Between group comparisons}

These comparisons address whether there are differences, with respect to hypothesized regions, between subject groups associated with different feedback conditions. The explicit hypothesis being tested was that the depressed patients would show abnormal blood flow in the regions associated with feedback in the normal group as described above. Where abnormalities were observed in these pre-specified regions, significance levels are determined using the analysis recommended by Worsley et al. (1996). Elsewhere a level of $P<0 \cdot 001$, uncorrected, is used, which is usually considered acceptable for exploratory analyses. As in the within-group analysis, contrasts between positive and negative feedback were not informative and are therefore excluded for clarity.

\section{Activity changes related to the presence compared to absence of feedback}

These comparisons represent main effects of feedback across the two tasks (see Table 2).
Changes in activity associated with presence compared with absence of feedback

When the activations associated with the presence of feedback were directly compared with the two subject groups, the controls showed significantly greater activation than depressed patients in the right $(P<0 \cdot 001)$ and left $(P<$ $0 \cdot 01$ ) medial caudate nuclei (Fig. $2 a$ ) and the tail of the caudate on the left. Using the analysis recommended by Worsley et al. (1996), the distance between the observed and the hypothesized focus in the right medial caudate was $5.6 \mathrm{~mm}$. The corrected $P$ value for the $Z$ score reported in Table 2 (employing a spherical region of $6 \mathrm{~mm}$ radius) would be $0 \cdot 0164$. For the left medial caudate focus, this value would be $0 \cdot 0374$. Adjusted $\mathrm{rCBF}$ values in the right medial caudate focus showed that there was lower activity overall in the depressed patients and that there were no changes associated with the different feedback conditions (Fig. 2b). There were no significant differences in deactivations associated with presence compared to absence of feedback between the two subject groups.

\section{Activity changes related to the modulation of} task performance by different types of feedback

These comparisons represent the interaction terms in the analysis (Table 3, Fig. 3).

Changes in activity associated with feedback compared with no feedback conditions in planning compared with guessing tasks

There were no significant differences between the two subject groups.

Changes in activity associated with feedback compared with no feedback conditions in guessing compared with planning tasks

When the subject groups were compared directly, 

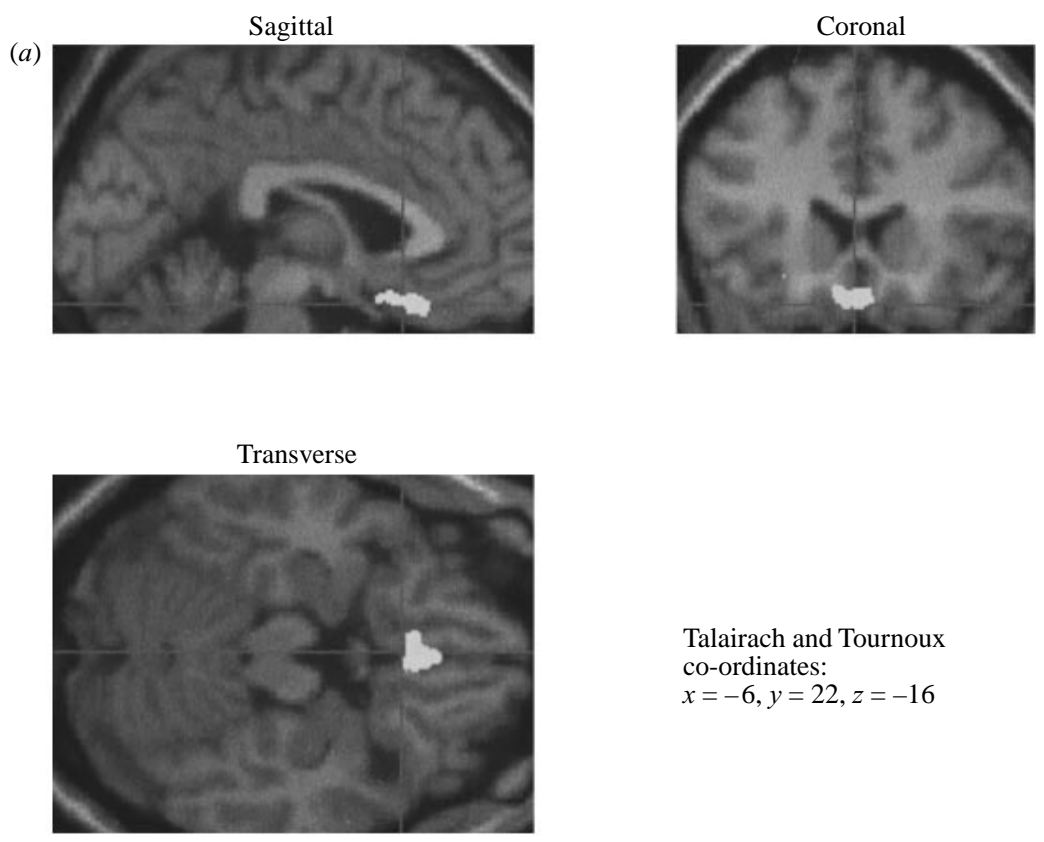

Talairach and Tournoux co-ordinates:

$x=-6, y=22, z=-16$

(b)

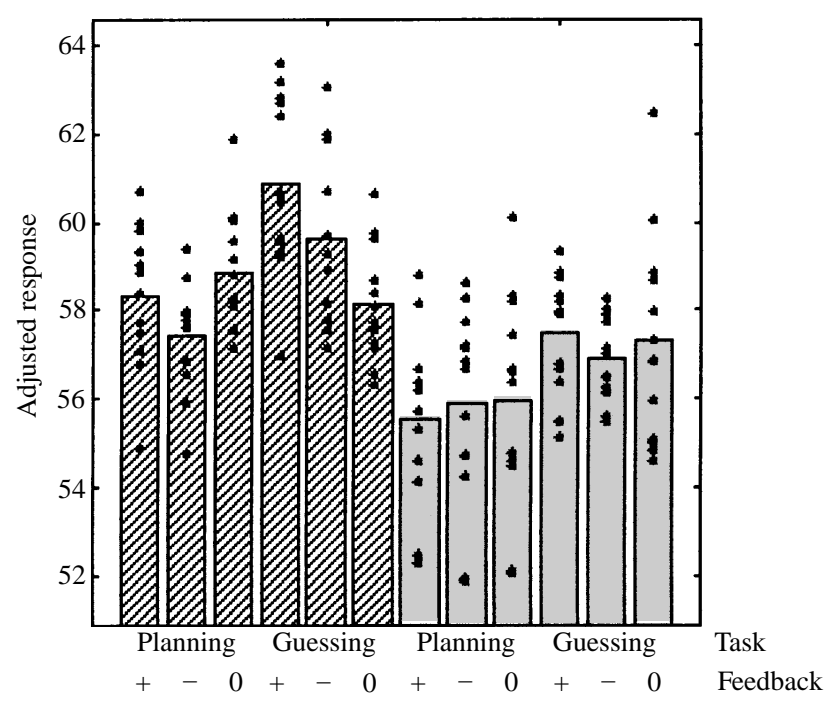

FIG. 3. Activations associated with the modulation of guessing compared to planning by presence compared to the absence of feedback, i.e. those areas more activated by the presence of feedback in the planning task than in the guessing task. (a) Shows the statistical parametric map of the $t$ statistic (after transformation to a $\operatorname{SPM}\{Z\}$ ) thresholded at $P<0.01$ rendered onto a standard MRI template and focused on the ventromedial orbitofrontal activation. (b) Shows absolute values of blood flow under the six experimental conditions in the ventromedial orbitofrontal cortex in the two subject groups ( $\square$, controls; 圆, patients).

significantly greater activation $(P<0 \cdot 01)$ was seen in the controls relative to the depressed patients in ventromedial orbitofrontal cortex (BA 25) (see Fig. 3a). Using the analysis recommended by Worsley et al. (1996), the distance between the observed and the hypothesized focus in the left ventromedial orbitofrontal cortex was $4.5 \mathrm{~mm}$. The corrected $P$ value for the $Z$ score reported in Table 3 (employing a spherical region of $4.5 \mathrm{~mm}$ radius) 


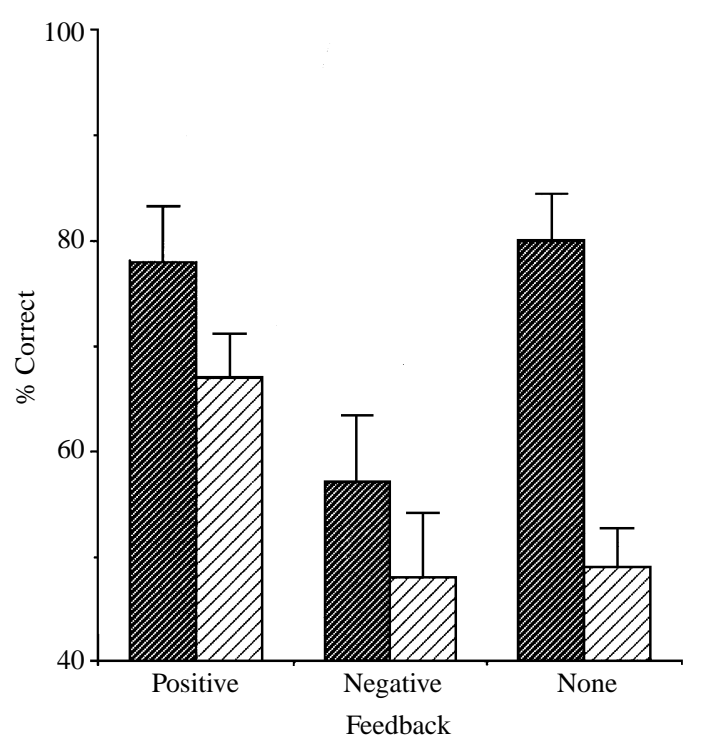

FIG. 4. Cognitive performance of the subjects (四, controls; $\square$, patients) on the planning task under the three feedback conditions. Mean percentage of problems solved correctly is shown. Bars show standard errors of the mean. Note that each condition was performed twice (1st and 2 nd presentation) in a counterbalanced order.

would be $0 \cdot 048$. For the right ventromedial orbitofrontal focus, this value would be 0.063 . Adjusted rCBF values (Fig. 3b) showed that in normal subjects blood flow was similar in the two feedback absent conditions but was higher in the feedback present conditions in the guessing task than in the planning task. Activity was lower overall in the depressed patients and the differential response associated with the different feedback conditions in the two tasks normal subjects was not evident.

\section{Performance data}

When subjects were debriefed after scanning, all the controls reported that they became aware the feedback was irrelevant to performance approximately halfway through the study session. By contrast, only a single depressed patient reported being aware that the feedback given was false. Fig. 4 shows the performance of both subject groups on the planning task in the different feedback conditions. Depressed patients performed less accurately overall, which is consistent with other neuropsychological studies using the Tower of London task (Beats et al. 1996; Elliott et al. 1996). Both groups performed less accurately in the negative feed- back condition than the positive condition. However, while the performance of control subjects on the neutral condition was similar to that on the positive condition, depressed patients receiving no feedback performed at a similar level to the negative feedback condition. On all conditions both subject groups performed substantially above chance levels $(17 \%)$ indicating that they were planning solutions rather than guessing. It is, therefore, not feasible that the differential task-related activations discussed below are due to depressed patients responding close to chance in the planning task. In fact, the overall percentages correct were not markedly different from those in our study of the Tower of London task performance with no time constraint (Elliott et al. 1997).

\section{DISCUSSION}

The main finding of this study was that while normal subjects showed a neural response in the medial caudate nucleus and the ventromedial orbitofrontal cortex as a function of level of performance feedback, this response was significantly attenuated in patients with unipolar depression. Activation within this network under feedback was modulated by task type in the normal subjects, with stronger activation seen during a guessing task than during a planning task. This profile of neural response to feedback, as a function of task type, was not seen in depressed patients.

In normal volunteers, caudate activation was seen in the feedback conditions of both tasks; it relates to the feedback itself, regardless of the cognitive processes that are being reinforced. This suggests that this activation may represent the emotive component of the response to feedback that is likely to be relatively independent of the task. In our earlier study (Elliott et al. 1992), we hypothesized that caudate activity might be associated with central representations of reward. This is compatible with the observation of Schultz et al. (1992) that neurons in the ventral striatum (which includes the medial caudate) of monkeys are activated before the expected delivery of reward. If the caudate signal is associated with anticipation of feedback rather than response to it, this may explain why there are no differences between activity in the positive and negative feedback conditions in 
either subject group. The present study suggests that depressed patients may have reduced activity associated with the expectation of reward. A decreased expectation of reward in depressed patients is consistent with the striking difference in the performance data for the two subject groups. Both groups solved fewer problems in the negative than the positive feedback condition. However, in the no feedback condition, normal controls solved as many problems as in the positive condition while depressed patients solved only as many as in the negative condition. In the absence of feedback therefore, normal subjects behave as if they are expecting success while depressed patients behave as if they are expecting failure. That is, in the absence of information to the contrary, the behaviour of patients suggests they may have significantly lower expectation of reward than controls. This account is consistent with psychological descriptions of depressed patients during reinforcementrelated tasks, for example, the finding of Hughes et al. (1985) that patients are less motivated than controls to respond for financial reward.

The activation of the ventromedial orbitofrontal cortex in normal controls was modulated by task type, with greater activation in a guessing compared to a planning task. This differential neural response was not seen in depressed patients. In our earlier paper, we proposed several explanations for the pattern of activity in the orbitofrontal cortex. One explanation accounted for the observed differences in terms of the perceived controllability of the tasks. In the planning task subjects believe that they have control over the type of feedback they receive; while in the guessing task they have no control. This difference in controllability may be related to the phenomenon of learned helplessness (Seligman, 1975) which behavioural theories have suggested may be associated with, or even model, depression (e.g. Abramson et al. 1978).

An alternative, but not unrelated, suggestion was that activity in the ventromedial orbitofrontal cortex was associated with an implicit requirement to process feedback across trials. In the planning task, each trial, incorporating problem, response and feedback, is a discrete and meaningful entity. In the guessing task however, feedback to a single trial is less meaningful; it is only when feedback is assimilated across a number of trials that subjects can assess their performance against chance. Furthermore, although subjects were told to guess at random, anecdotal evidence of behaviour in response to chance determined events would predict that they probably monitored performance and chose their responses in the light of previously rewarded responses. Certainly, a consideration of the actual sequences produced by both subject groups in the guessing task, suggests they are not genuinely random, for example, no subject ever made the same response on successive trials. In the guessing task, the feedback is the only information the subjects have about performance and the orbitofrontal component of the response may thus represent this informational aspect of response to feedback.

An involvement of orbitofrontal cortex in acquiring and monitoring stimulus-reinforcement relations has been reported in studies of monkeys (Thorpe et al. 1983; Dias et al. 1996) and patients with orbitofrontal lesions (Bechara et al. 1994, 1996; Rolls et al. 1994). Depressed patients failed to show the normal activation of the orbitofrontal cortex and this may be related to an impaired ability to process response-outcome associations or reward contingencies across trials. This suggestion would be compatible with the observation that while all the control subjects realised the feedback was false by the end of the experiment, only one of the depressed patients did so, and even he was unsure. Failure to process response-outcome associations may contribute to learned helplessness in depression. Impaired learning about correlations between behaviour and positive outcomes is a crucial component in determining the extent to which a situation can be controlled.

In this study, depressed patients are underresponsive to feedback compared to controls in terms of neural response. Our recent neuropsychological study (Elliott et al. 1997) suggested that compared with controls, depressed patients fail to show an elevated probability of correctly solving a problem directly after a failure. That is, controls respond to failure by improving performance to increase their chance of success on the subsequent problem. Depressed patients do not. This could actually be characterized as an undersensitivity to negative feedback and may depend on a combination of cognitive and emotional factors. Thus, patients may be less 
able than controls to use the information conveyed by feedback to monitor and correct subsequent performance. They may also show impaired motivation to respond for positive feedback. It is not entirely clear how this account relates to the behavioural data presented in this study. We have proposed that controls respond to negative feedback by improving performance while patients do not. In fact the controls in this study performed worse under negative feedback than when no feedback was given. However, a crucial point is that these data are for a task where the feedback is not related to performance so that no amount of trying to improve performance has the desired outcome. In fact, in the negative feedback condition, the feedback may misleadingly encourage control subjects to change successful performance strategies, resulting in an increased error rate. Depressed patients perform no worse in the negative than the neutral condition, perhaps implying that this inappropriate monitoring does not occur. In cognitive terms, the situation in this study is different from the usual one where feedback relates to performance. This is an issue that would merit clarification by a neuropsychological study but is not central to the neurobiological argument of the present study.

The account of the neuropsychological finding as a reduced ability of depressed patients to improve performance in the face of negative feedback is more consistent with the neural hypoactivity in response to feedback seen in the present study. It is also consistent with the many animal models of depression that have used decreased responding for reward as a critical analogue of human depression (e.g. Zacharko \& Anisman, 1991; Moreau et al. 1994). Many cognitive and behavioural accounts of depression also stress a role for reduced rewardrelated behaviour. Layne (1980) explicitly argued that depression 'consists' of low reward motivation while other accounts have also proposed reduction in experience of positive reinforcement as an important factor (Lewinsohn et al. 1979).

A striking finding of this study was that within the structures activated in the presence of performance feedback, there were no differences associated with different types of feedback (positive compared to negative). If the activations in the medial caudate or orbitofrontal cortex were specific to the actual experience of reward, this would not have been the case. Instead, we have argued that the ventral striatal activation may be associated with the expectation of reward and the orbitofrontal activation with learning about response-outcome relations. Reduced activation in the depressed patients could thus be interpreted as related to reduced expectation of reward and impaired ability to learn about stimulus-reward relations rather than an impaired response to reward per se.

Many previous functional imaging studies have suggested that depression is associated with reduced activity in the anterior cingulate and medial prefrontal cortex (George et al. 1993; Goodwin et al. 1993; Dolan et al. 1994). The present study did not show cingulate abnormality but demonstrated a much more ventral medial prefrontal abnormality. In this version of the paradigm, task performance was not associated with cingulate activation in the normal subjects. We argued that this may be due to this structure also being activated in the guessing task, which was more demanding than the original control task. It may be, therefore, that cingulate activity was indeed lower in depressed patients but this was not revealed by the contrasts used in the present study. We suggest that medial prefrontal dysfunction in depression may be relatively extensive with the focus of the identified dysfunction depending on the precise study conditions. Both the present and our earlier study identified reduced activation of the caudate nucleus, an area also implicated in other functional imaging studies of depression (Buchsbaum et al. 1992; Drevets et al. 1992). Striatal pathophysiology may compromise function in cortico-striatal loops that feed back to the orbital, cingulate and medial prefrontal cortex (Alexander et al. 1986). It has been suggested that depression is associated with disrupted function within frontostriatal loops (Robbins et al. 1992) and our functional imaging studies suggest that the prefrontal expression of this abnormality may depend on the specific cognitive requirements imposed on patients.

It should be noted that the patients in this study were taking antidepressant medication at the time of scanning and it will obviously be important to replicate these results in unmedicated patients in order to rule out possible confounding effects of treatment. There is some 
recent evidence suggesting that abnormalities in medial prefrontal areas may be to an extent independent of medication effects. For example George et al. (1997) demonstrated blunted response in the anterior cingulate of unmedicated depressed patients performing the Stroop task. Mann et al. (1996) showed that a group of drug-free depressed patients failed to show the normal increase in metabolism in medial prefrontal regions in response to the serotonergic drug fenfluramine. There is also evidence though, that antidepressant treatment may have a direct effect on these areas. Mayberg et al. (1997) reported that SSRI treatment decreased ventromedial frontal glucose metabolism and therefore it is possible that some of the overall reduction in ventromedial blood flow in the present study is a drug effect. However, only half the patients were taking selective serotonin reuptake inhibitors and the blood flow pattern we observed was consistent across patients. Also, although a drug effect may explain the lower overall blood flow, it cannot necessarily account for the lack of condition specific effects. These issues can only fully be clarified by further studies in unmedicated patients and it is important to note the caveat imposed by this limitation of the present data.

In conclusion, the results of this study suggest that response to feedback in patients with unipolar depression is associated with attenuation of activation within a network including the medial caudate nucleus and ventrolateral orbitofrontal cortex. Modulation of feedback related activity by task type suggests that different components of this network subserve subtly different functions in the response. We have proposed that these neural abnormalities in depressed patients reflect a decreased expectation of reward and an impaired ability to learn response-outcome contingencies, possibly contributing to learned helplessness. While these results cannot be regarded as conclusive, they suggest that a crucial link between affect and cognitive impairment in depression may depend on a clearly circumscribed neural substrate.

R. J. Dolan is supported by the Wellcome Trust. This research was funded in part by a Wellcome Trust Programme Grant to Drs Robbins, Sahakian, Everitt and Roberts. We are grateful to Karl Friston for statistical help, to Trevor Robbins, Chris Frith, Cathy
Price and an anonymous reviewer for comments and discussion and to Fionnuala Murphy for help with escorting the patients.

\section{REFERENCES}

Abramson, L. Y., Seligman, M. E. P. \& Teasdale, J. (1978). Learned helplessness in humans: critique and reformulation. Journal of Abnormal Psychology 87, 49-74.

Alexander, G. E., DeLong, M. \& Strick, P. E. (1986). Parallel organization of functionally segregated circuits linking basal ganglia and cortex. Annual Review of Neuroscience 9 357-381.

American Psychiatric Association (1994). Diagnostic and Statistical Manual of Mental Disorders (DSM-IV). APA: Washington, DC.

Austin, M.-P., Ross, M., Murray, C., O'Carroll, R. E., Ebmeier, K. P. \& Goodwin, G. M. (1992). Cognitive function in major depression. Journal of Affective Disorders 25, 21-30.

Beats, B. C., Sahakian, B. J. \& Levy, R. (1996). Cognitive performance in tests sensitive to frontal lobe dysfunction in the elderly depressed. Psychological Medicine 26, 591-603.

Bechara, A., Damasio, A. R., Damasio, H. \& Anderson, S. W. (1994). Insensitivity to future consequences following damage to human prefrontal cortex. Cognition 50, 7-15.

Bechara, A., Tranel, D., Damasio, H. \& Damasio, A. R. (1996). Failure to respond autonomically to anticipated future outcomes following damage to the prefrontal cortex Cerebral Cortex 6, 215-25.

Beck, A. T. (1967). Depression: Clinical, Experimental and Theoretical Aspects Harper and Row: New York.

Bench, C. J., Friston, K. J., Brown, R. G., Scott, L. C., Frackowiak, R. S. J. \& Dolan, R. J. (1992). The anatomy of melancholia - focal abnormalities of cerebral blood flow in major depression. Psychological Medicine 22, 607-615.

Brown, R. G., Scott, L. C., C. J. \& Dolan, R. J. (1994). Cognitive function in depression: its relationship to the presence and severity of intellectual decline. Psychological Medicine 24, 829-847.

Buchsbaum, M., Wu, J., DeLisi, L., Holcomb, H., Kessler, R., Johnson, J., King, A., Hazlett, E., Langston, K. \& Post, R. (1986). Fronal cortex and basal ganglia metabolic rates assessed by positron emission tomography with $\left[{ }^{18} \mathrm{~F}\right] 2$-deoxyglucose in affective illness. Journal of Affective Disorders 10, 137-152.

Buchsbaum, M. S., Haier, R. J., Potkin, S. G., Nuechterlein, K., Bracha, H. S., Katz, M., Lohr, J., Wu, J., Lottenberg, S., Jerabek, P. A., Trenary, M. M., Tafalla, R., Reynolds, C. \& Bunney, W. E. (1992). Frontostriatal disorder of cerebral metabolism in nevermediated schizophrenics. Archives General Psychiatry 49, 935-942.

Cohen, R., Semple, W., Gross, M., Nordahl, T., King, A. C., Pickar,

D. \& Post, R. (1989). Evidence for common alterations in cerebral glucose metabolism in major affective disorders and schizophrenia. Neuropsychopharmology 2, 241-254.

Dias, R., Robbins, T. W. \& Roberts, A. C. (1996). Dissociation in prefrontal cortex of affective and attentional shifts. Nature $\mathbf{3 8 0}$, 69-72.

Dolan, R. J., Bench, C. J., Brown, R. G., Scott, L. C. \& Frackowiak, R. S. J. (1994). Neuropsychological dysfunction in depression: the relationship to regional cerebral blood flow. Psychological Medicine 24, 849-857.

Drevets, W. C., Videen, T. O., Price, J. L., Preskorn, S. H., Carmichael S. T. \& Raichle, M. E. (1992). A functional anatomical study of unipolar depression. Journal of Neuroscience 12, 3628-3641.

Drevets, W. C., Price, J. L., Simpson, J. R., Todd, R. D., Reich, T., Vannier, M. \& Raichle, M. E. (1997). Subgenual prefrontal cortex abnormalities in mood disorders. Nature 386, 824-827.

Duvernoy, H. M. (1991). The Human Brain: Surface, Threedimensional Sectional Anatomy and MRI. Springer Verlag: New York. 
Elliott, R., Sahakian, B. J., McKay, A. P., Herrod, J. J., Robbins, T. W. \& Paykel, E. S. (1996). Neuropsychological impairments in unipolar depression: the role of perceived failure on subsequent performance. Psychological Medicine 26, 975-989.

Elliott, R., Baker, S. C., Rogers, R. D., O’Leary, D. A., Paykel, E. S., Frith, C. D., Dolan, R. J. \& Sahakian, B. J. (1997). Prefronta dysfunction in depressed patients performing a planning task: a study using positron emission tomography. Psychological Medicine 27, 931-9.

Friston, K. J., Holmes, A. P., Worsley, K. J., Poline, J.-B., Frith, C. D. \& Frackowiak, R. S. J. (1995). Statistical parametric maps in functional imaging: a general approach. Human Brain Mapping 2 189-210.

George, M. S., Ketter, T. A. \& Post, R. M. (1993). SPECT and PET in mood disorders. J. Clinical Psychiatry 54 (suppl.), 6-13.

George, M. S., Ketter, T. A., Parekh, P. I., Rosinsky, N., Ring, H. A., Pazzaglia, P. J., Maragell, L. B., Callahan, A. M. \& Post, H. M. (1997). Blunted left cingulate activation in mood disorder subjects during a response interference task (the Stroop). Journal of Neuropsychiatry and Clinical Neuroscience 9, 55-63.

Goodwin, G. M., Austin, M.-P., Dougall, N., Ross, M., Murray, C., O'Carroll, R. E., Moffoot, A., Prentice, N. \& Ebmeier, K. P. (1993). State changes in brain activity shown by the uptake of $99 \mathrm{~m}$ Tc-exametazime with single photon emission tomography in major depression before and after treatment. Journal of Affective Disorders 29, 243-253.

Hamilton, M. (1960). A rating scale for depression. Journal of Neurology, Neurosurgery and Psychiatry 23, 56-62.

Henriques, J. B., Glowacki, J. M. \& Davidson, R. J. (1994). Reward fails to alter response bias in depression. Journal of Abnormal Psychology 103, 460-466.

Hughes, J. R., Pleasants, C. N. \& Pickens, R. W. (1985). Measurement of reinforcement in depression: a pilot study. Journal of Behavioural Therapy and Experimental Psychiatry 16, 231-236.

Layne, C. (1980). Motivational deficit in depression: people's expectations $\times$ outcomes' impacts. Journal of Clinical Psychology 36, 647-652.

Lewinsohn, P. M. (1974). A behavioural approach to depression. In The Psychology of Depression: Contemporary Theory and Research (ed. R. J. Friedman and M. M. Katz), pp. 157-185. Winston/ Wiley: New York.

Lewinsohn, P. M., Youngren, M. A. \& Grosscup, S. J. (1979). Reinforcement and depression. In The Psychobiology of Depressive Disorders (ed. R. A. Depue), pp. 291-316. Academic Press: New York.

Mann, J. J., Malone, K. M., Diehl, D. J., Perel, J., Cooper, T. B. \& Mintun, M. A. (1996). Demonstration in vivo of reduced serotonin responsivity in the brains of untreated depressed patients. American Journal of Psychiatry 153, 174-182.

Mayberg, H. S., Lewis, P. J., Regenold, W. \& Wagner, H. N. (1994). Paralimbic hypoperfusion in unipolar depression. Journal of Nuclear Medicine 35, 929-934.
Mayberg, H. S., Brannan, S. K., Mahurin, R. K., Jerabek, P. A., Brickman, J. S., Tekell, J. L., Silva, J. A., McGinnis, S., Glass, T. G., Martin, C. C. \& Fox, P. T. (1997). Cingulate function in depression: a potential predictor of treatment response. Neuroreport $\mathbf{8}, 1057-1061$.

Montgomery, S. A. \& Åsberg, M. (1979). A new depression scale designed to be sensitive to change. British Journal of Psychiatry 134, 382-389.

Moreau, J. L., Bourson, A., Jenck, F., Martin, J. R. \& Mortas, P. (1994). Curative effects of the atypical antidepressant mianserin in the chronic mild stress-induced anhedonia model of depression. Journal of Psychiatry and Neuroscience 19, 51-56.

Owen, A. M., Sahakian, B. J., Hodges, J. R., Summers, B. A., Polkey, C. E. \& Robbins, T. W. (1995). Dopamine-dependent frontostriatal planning deficits in early Parkinson's disease. Neuropsychology 9, 126-140.

Robbins, T. W., Joyce, E. M. \& Sahakian, B. J. (1992). Neuropsychology and Neuroimaging of affective disorders. In Handbook of Affective Disorders (ed. E. S. Paykell), pp. 289-309. Churchill Livingstone: London.

Rolls, E. T., Hornak, J., Wade, D. \& McGrath, J. (1994). Emotion related learning in patients with social and emotional changes associated with frontal lobe damage. Journal of Neurology, Neurosurgery and Psychiatry 57, 1518-24.

Schultz, W., Apicella, P., Scarnati, E. \& Ljungberg, T. (1992). Neuronal activity in monkey ventral striatum related to the expectation of reward. Journal of Neuroscience 12, 4595-4610.

Seligman, M. E. P. (1975). Helplessness: On Depression, Development and Death. Freeman: San Francisco.

Shallice, T. (1982). Specific impairments in planning. Philosophical Transactions of the Royal Society B298, 199-209.

Talairach, J. \& Tournoux, P. (1988). Coplanar Stereotactic Atlas of the Human Brain. George Thieme Verlag: Stuttgart.

Teasdale, J. D. (1988). Cognitive vulnerability to persistent depression. Cognition and Emotion 2, 247-274.

Thorpe, S. J., Rolls, E. T. \& Maddison, S. (1983). The orbitofrontal cortex: neuronal activity in the behaving monkey. Experimental Brain Research 49, 93-115.

Weingartner, H., Cohen, R. M., Martello, J. D. \& Gerdt, C. (1981). Cognitive processes in depression. Archives of General Psychiatry 38, $42-47$.

Wener, A. E. \& Rehm, L. P. (1975). Depressive affect: a test of behavioral hypotheses. Journal of Abnormal Psychology 84, 221-227.

Worsley, K. J., Marrett, S., Neelin, P., VAndal, A. C., Friston, K. J. \& Evans, A. C. (1996). A unified statistical approach for determining significant signals in images of cerebral activation. Human Brain mapping 4, 58-73.

Zacharko, R. M. \& Anisman, H. (1991). Stressor-induced anhedonia in the mesocorticolimbic system. Neuroscience and Biobehavioural Review 15, 391-405. 